\title{
Le Rayon vert de Jules Verne. La confrontation ironique des discours météorologiques
}

\author{
Jules Verne's Le Rayon vert. \\ The ironical confrontation of meteorological discourses
}

\author{
Karin Becker \\ Universität Münster \\ kabecker@uni-muenster.de
}

\begin{abstract}
Jules Verne's Le Rayon vert (1882) is at the same time a scientific novel and a love story, a repertory of knowledge and a criticism of clichés. Telling the amusing attempts of a little group of travelers that tries in vain to catch sight of the "green ray" - the last impression of sunset - on the horizon from the Scottish coast, the author confronts in an ironical way different stereotypical discourses about weather phenomena which coexist in the bourgeois society of the late $19^{\text {th }}$ century. Verne mocks equally scientific, folkloristic and romantic ideas about meteorological observations and uses these opposite explications of the mysteries of nature to characterize his protagonists and to develop the suspense of the narration.
\end{abstract}

Keywords: Jules Verne, scientific novel, romanticism, meteorology, love story

À Anouchka Vasak

Dans Le Rayon vert ${ }^{1}$, son seul véritable roman d'amour ${ }^{2}$, Jules Verne oppose, dans une visée largement caricaturale, plusieurs attitudes stéréotypées de ses contemporains face aux phénomènes atmosphériques. Dans cette histoire amusante d'une jeune

\footnotetext{
${ }^{1}$ Le roman est rédigé à partir de 1881, publié en feuilleton dans Le Temps de mai à juin 1882, et finalement édité chez Hetzel en juillet 1882.

${ }^{2}$ Dans une lettre adressée à son éditeur Hetzel, Jules Verne écrit : « Je suis très maladroit à exprimer des sentiments d'amour. Ce mot-là seul ,,amour” m'effraye à écrire » (cité d'après Verne, 1973, p. 128).
} 
fille qui cherche obstinément à surprendre sur l'horizon marin écossais le dernier rayon magique, de couleur verte, émis par le soleil couchant, cette mise en regard ironique des différents discours météorologiques (scientifique, folklorique, romantique, etc.) est très explicite, car les interprétations contraires de l'état du ciel servent à caractériser les protagonistes et à structurer le déroulement de l'intrigue. Pourtant, cette imbrication complexe des propos opposés sur les " météores $\|^{3}$ - une orchestration polyphonique d'ailleurs typique de l'écriture vernienne - n'a pas encore intéressé la critique, qui a peu étudié ce roman exceptionnel et ambivalent ${ }^{4}$ et qui néglige plus généralement le thème de la pluie et du beau temps ${ }^{5}$ pourtant omniprésent dans l'œuvre de l'inventeur du « roman scientifique ».

Le rayon vert, motif central de la narration, représente un photométéore pratiquement inconnu en 1882, lequel doit son nom et sa célébrité au roman de Jules Verne, sans qu'il soit possible d'identifier une source précise qui l'aurait inspiré (Frappa, 2019 , p. 77-89) ${ }^{6}$. Ce phénomène optique qui s'explique de nos jours par la conjugaison de la réfraction différentielle et de l'absorption atmosphérique, ne sera analysé par les scientifiques que suite à la publication du récit, de sorte que son statut reste énigmatique chez Verne, suscitant les exégèses les plus diverses. Dès le début du roman, cette ambiguité du rayon est introduite par un témoignage paradoxal, proposant deux approches antithétiques. Une « citation » insérée dans le troisième chapitre consiste en un article (fictif) attribué au journal londonien Morning Post, en principe un garant de l'authenticité et du sérieux de l'information. Le journaliste anonyme conseille aux lecteurs de chercher à épier « le phénomène qui se produit à l'instant précis où l'astre radieux lance son dernier rayon, si le ciel, dégagé de brumes, est alors d'une pureté parfaite », en insistant sur le caractère extraordinaire de la vision, car « ce ne sera pas comme on pourrait le croire, un rayon rouge qui viendra frapper la rétine de votre œil, ce sera un rayon „vert” »(Verne, 2005, p. 31). La lecture de ces lignes incite Helena

${ }^{3}$ Le terme, dérivé du grec, désigne tout phénomène se produisant dans l'atmosphère : son étymologie renvoie à « ce qui est en haut, ou monte dans les airs, mais aussi ce qui est en perpétuel changement ». Les météorologues distinguent les hydrométéores (pluie, neige, grêle, brouillard, etc.), les lithométéores (sables, poussières, etc.), les photométéores (arc-en-ciel, halo, aurore boréale, etc.) et les électrométéores (éclair, tonnerre, etc.), en écartant paradoxalement les vents et les nuages. Cf. Belleguic \& Vasak, 2013, p. 1-26. Nous entendrons ici le mot «météorologie » avec Anouchka Vasak moins comme une science naturelle que dans le sens très global de « discours sur les météores » (Vasak, 2008, p. 406, note $2 ;$ p. 488).

${ }^{4}$ La bibliographie n'est pas longue : Chevrier, 1999, p. 25-29 ; Derivery, 1994, p. 9-43 ; Le Scanff, 2007, p. 249-262 ; Picot, 1992, p. 57-83 ; Porcq, 1990, p. 40-44 ; Pourvoyeur, 1990, p. 33-37 ; Raymond, 1994, p. 101-116; Sadaune, 1999, p. 14-21.

${ }^{5}$ À notre connaissance, il n'existe que deux articles sur le sujet : Robin, 2008, p. 57-64 ; Le Lay \& Tirard, 2011, p. 195-203.

${ }^{6}$ Voir aussi les sites de Jacques Crovisier https://lesia.obspm.fr/perso/jacques-crovisier/JV/verne RV.html et de Andrew T. Young, https://aty.sdsu.edu/ (consultés le 24 mars 2020). Quant à Klaus Bonn (Bonn, 2014), il se réfère, lui aussi à Andrew T. Young (Young, 1998, p. 189) ainsi qu'à Fred Schaff (Schaff, 2006, p. 30-34). 
Campbell à se lancer à la poursuite de ce météore fascinant, qui fait donc figure de moteur de l'intrigue.

Or, c'est moins l'évocation de l'aspect physique du phénomène qui la «passionne » que le passage final de l'article, qui change complètement de tonalité, abandonnant le rapport neutre pour un hymne lyrique, chantant la beauté de ce rayon fabuleux. En reprenant les topoï romantiques d'une esthétique du sublime et de l'indicible (Saint Girons, 2005) ${ }^{7}$, l'auteur s'extasie sur le pouvoir mystérieux de ce météore qui serait « d'un vert merveilleux, d'un vert qu'aucun peintre ne peut obtenir sur sa palette, d'un vert dont la nature, ni dans la teinte si variée des végétaux, ni dans la couleur des mers les plus limpides, n'a jamais reproduit la nuance! S'il y a du vert dans le Paradis, ce ne peut être que ce vert-là, qui est, sans doute, le vrai vert de l'Espérance ! » (Verne, 2005, p. 32). Cette part du rêve et de l'imaginaire, la tendance au merveilleux, qui accompagne toujours la dimension scientifique des œuvres verniennes ${ }^{8}$, dépasse donc la description objective de l'élément naturel, pour en faire un signe (sur-)chargé de sens, un chiffre du grand livre de la nature, qui comprend le météore dans la valeur - esthétique, morale, théologique et ontologique - qu'il présente pour l'existence humaine ${ }^{9}$. Et ce n'est pas un hasard si Verne choisit la majuscule, faisant du « Rayon-Vert» une entité autonome, voire un principe cosmologique.

Ces deux versants du discours météorologique, combinés dans un «document » apparemment historique, s'avèrent finalement complémentaires, reflétant, tel un miroir déformant, deux postures-clichés répandues dans la société bourgeoise de l'époque. Ils fusionnent dans la personne de la jeune héroïne, dont un portrait psychologique distingue « deux êtres » : «Le „moi” de Miss Campbell, c'était l'être sérieux, réfléchi [...]. „L'autre”, c'était l'être romanesque, un peu enclin aux superstitions » (Verne, 2005, p. 30). En fait, tous les deux regards portés sur le ciel font l'objet d'une moquerie subtile et amusée. La première partie de l'article, adressée aux « amateurs de curiosités physiques », est un pastiche du style enjoué des vulgarisateurs scientifiques, avec ses questions suggestives et ses réponses souvent trop simples (« Avezvous quelque fois observé le soleil [...] ? Oui ! sans doute. [...] Mais avez-vous remarqué le phénomène $[\ldots]$ ? Non ! peut-être. Eh bien, $[\ldots]$ ce ne sera pas $[\ldots]$, ce sera $[\ldots] »$, Verne, 2005 , p. 31). Cette prise de distance ironique par rapport aux

${ }^{7}$ Le terme « sublime » évoque une nature grandiose et incommensurable, qui dépasse l'expérience quotidienne et provoque l'étonnement et l'admiration, mais aussi un sentiment d'impuissance (« delightful horror », Burke, 1792, p. 109, 219), savouré en pleine conscience et esthétisé par la littérature et les beaux-arts.

${ }^{8}$ Voir Roboly, 2005, p. 168-175 ; Vierne, 2005, p. 299-306 ; Compère, 2013 ; Dupuy, 2013, p. 41-60. La conception de la « science » chez Jules Verne a ses racines dans la philosophie naturelle de la fin des Lumières et du romantisme, dont l'approche holistique (avant la séparation des « deux cultures » au XIX ${ }^{\mathrm{e}}$ siècle et la naissance du positivisme) inclut le rapport que l'homme entretient avec la nature. Cf. Dehs, 2005, p. 247-265, et Michel, 1980, p. 117-123.

${ }^{9}$ Pour les différentes interprétations (scientifiques, religieuses, anthropologiques, symboliques...) du temps qu'il fait en général, voir Becker, 2012a, p. 15-56. 
ouvrages grand public est d'autant plus remarquable que Jules Verne lui-même, loin d'être un scientifique, puise ses idées et ses arguments dans ce genre de littérature et contribue de son côté à cette veine de publications. Or, le même questionnement espiègle mine, d'une façon subreptice, les allusions aux belles-lettres, dont fourmille l'œuvre vernienne ${ }^{10}$, ici la référence aux adeptes de l'enthousiasme romantique, de sorte que ce premier échantillon « journalistique » d'une écriture météorologique en combine déjà plusieurs facettes conventionnelles.

Aussitôt après cet article protéiforme « tiré » d'un quotidien, le romancier enchaîne un troisième discours, folklorique, populaire, relevant d'une tradition orale, remontant aux temps archaïques et suggérant une puissance magique :

[...] ce Rayon-Vert se rapportait à une vieille légende [...], légende inexpliquée entre tant d'autres, nées au pays des Highlands, et qui affirme ceci : c'est que ce rayon a pour vertu de faire que celui qui l'a vu ne peut plus se tromper dans les choses de sentiment; c'est que son apparition détruit illusions et mensonges ; c'est que celui qui a été assez heureux pour l'apercevoir une fois, voit clair dans son cœur et dans celui des autres (Verne, 2005, p. 32).

Bien entendu, cette « légende » de la vieille Écosse est, elle aussi, une invention originale de Jules Verne, dont l'imagination est profondément marquée par cette culture ancienne, peut-être en raison de son ascendance maternelle, mais aussi suite aux deux visites qu'il effectua dans le pays en 1859 et en 1879 . Cette référence à la mythologie nordique reste un thème récurrent du récit, grâce aux constantes évocations des « poésies ossianesques » (Verne, 2005, p. 253, cf. p. 153, 175, 219, 224, 227, etc.) et à une vision panthéiste du paysage des Hébrides, où des « êtres surnaturels », des « déités [...] immatérielles », des « poétiques fantômes, échappés aux livres des Sagas » semblent surgir à tout moment, de sorte que Helena soupçonne dans le rayon vert « l'écharpe de quelque Valkyrie » (Verne, 2005, p. 179).

À travers le prisme romantique, l'exégèse mythologique du rayon vert insiste sur la dimension visuelle, sur le regard individuel et subjectif. Ainsi, l'observation du phénomène se transforme métaphoriquement en une sorte de seconde vue, fonctionnalisée sur le plan de la vie sociale et plus concrètement sur celui de l'amour. Car c'est finalement ce sens symbolique attribué au météore qui pousse Helena à la quête du bonheur sentimental, car elle refuse d'accepter le mari proposé par ses oncles tant qu'elle n'aura pas vu la «vérité » révélée par le rayon, prometteur d'une union heureuse. Ainsi, ce singulier « roman de l'œil » de Jules Verne banalise d'une manière plaisante le style grandiloquent de l'interprétation métaphysique de la nature, en réduisant son sens supérieur à la réalisation toute pratique d'un projet de mariage, qui constitue le véritable but de la « chasse au météore ». En conséquence logique, la

${ }^{10}$ Sur le vaste réseau intertextuel voir Evans, 1996, p. 171-187. Les écrivains mentionnés (ou présents en filigrane) dans le Rayon vert sont Michelet, Musset, Dickens, Wordsworth, et surtout Scott et «Ossian » (i.e. Macpherson). 
dernière scène de la narration montre Helena et son amoureux, Olivier Sinclair, face à l'effet optique tant poursuivi, mais plongés dans leurs regards réciproques : «Olivier et Helena n'avaient rien vu du phénomène, qui venait enfin d'apparaître après tant d'infructueuses observations ! » (Verne, 2005, p. 258-259). La quête du rayon vert s'avère donc « une fable au second degré » au fond érotique, qui se termine par « l'accomplissement de sa fonction » (Derivery, 1994, p. 18-19) ${ }^{11}$.

La longue recherche du meilleur observatoire possible, se traduisant par un itinéraire menant toujours plus avant vers le Nord sur la côte atlantique écossaise ${ }^{12}$, constitue un parcours difficile dont les étapes rythment le développement de l'histoire. Le suspense résulte d'une série d'innombrables obstacles, d'échecs consécutifs et de tentatives renouvelées pour accéder à la vision du phénomène. Ainsi, la course est semée d'une succession de frustrations et de relances, et les difficultés rencontrées font figure d'épreuves à surmonter, lesquelles révèlent le caractère héroïque des protagonistes - le courage infaillible d'Helena surtout, qui entraîne un petit groupe de voyageurs - et lesquelles confèrent une portée initiatique (Vierne, 1973) à cette quête acharnée (« cette fantaisie devint une idée fixe. [...] Cela tournait à l'état d'obsession », Verne, 2005, p. 142). Or, la grande majorité des adversités à combattre consiste dans les caprices du ciel, dans les divers aléas météorologiques, qui voilent l'horizon où le miracle est censé se produire ${ }^{13}$. Ces intempéries incessantes qui jalonnent les péripéties contrarient donc fortement les plans de la petite compagnie : sur le plan narratologique, les manifestations atmosphériques constituent des agents majeurs, des antagonistes doués d'une volonté propre, qui représentent pour les participants de l'expédition une force majeure, sinon une « fatalité » (Verne, 2005, p. 129). Ainsi naît un conflit entre les «bons » et les « mauvais » météores, entre un ciel bénéfique et néfaste pour la réussite de l'entreprise entamée.

Car le rayon vert et les autres météores (les hydrométéores surtout), dont l'irruption empêche l'exécution du projet d'observation, présentent en fait des caractéristiques antinomiques. Le rayon, tout en étant un photométéore, relève en même temps de l'astronomie, d'une science « exacte » qui étudie les corps célestes dans leur régularité mécanique et bien calculable et dont l'importance pour l'œuvre de Jules Verne

${ }^{11}$ Le rayon vert relève ainsi du symbolisme du feu et de la flamme, à la fois pour la valeur de révélation et de moyen de connaissance et pour l'expressivité émotionnelle, suggérant des passions fortes et notamment le désir sexuel. Cf. Becker, 2016, p. 9-28.

${ }^{12}$ Voir les cartes publiées dans le roman lui-même (Verne, 2005, p. 80) ainsi que dans Sadaune, 2004, p. 64.

${ }^{13}$ En fait, selon le météorologiste Louis Dufour, « le rayon vert est une coloration à dominante verte et de courte durée, souvent sous forme d'un éclat bref, qui se produit lorsque l'extrême bord supérieur du soleil disparaît sous l'horizon. Ce photométéore s'observe lorsque l'horizon est très pur et le disque solaire ni rouge ni orangé, mais très éclatant. [...]. La formation du rayon vert dépend de tant de facteurs qu'il n'est pas surprenant que son apparition semble capricieuse. Entrent en effet en jeu dans sa formation non seulement sa réfraction, sa dispersion et l'absorption de la lumière du soleil, mais aussi la structure et la composition de l'atmosphère » (Dufour, 1982, p. 122-123). C'est nous qui soulignons. 


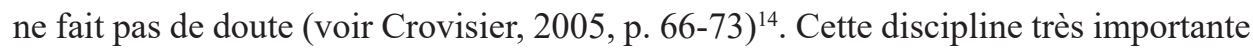
à l'âge du positivisme promet la validité incontestable d'un système de règles, c'està-dire la précision, l'abstraction, la conceptualisation, la classification et la rationalisation - critères sur lesquels comptent Helena et ses proches pour attendre, à une heure précise et à un endroit défini, l'astre solaire à un moment bien déterminé de sa trajectoire («Astronomiquement, le soleil, à cette époque de l'année et pour cette latitude, devait se coucher à sept heures cinquante-quatre minutes, précisément dans la direction de la pointe Ardanalish », Verne, 2005, p. 101). L'astronomie constitue la science « de l'espace de l'abstraite mathesis, de la géométrie analytique, de la mécanique céleste, de la physique générale des solides, des corps inertes, du calcul infinitésimal » (Belleguic, 2003, p. 23) - un royaume de l'ordre et de la continuité, pourtant toujours assujetti aux conditions atmosphériques qui peuvent, souvent brusquement, déranger la contemplation de la transparence initiale de la voûte étoilée.

La météorologie, en revanche, «n'est pas une science, au sens exact, puisqu'elle „ne" traite „que" de ces objets instables, mal définis, imprévisibles, que sont les météores » (Vasak, 2008, p. 484), des " phénomènes instables, fluides, volatils, subtils et difficiles, [...] privés de régularités simples, fourmillant d'aléas » (Serres, 1994, p. 97). En outre, les deux disciplines diffèrent par leur conception du temps : à la temporalité linéaire, chronologique de l'astronomie (« le temps qui passe ») s'oppose le mouvement du temps qui coule, le cycle du temps de la nature, plus irrégulier et difficile à prédire (« le temps qu'il fait »). L'atmosphère est fugace et en éternelle transformation, d'un désordre constitutif et d'une empirie contingente. Ainsi, les savantes conjectures des personnages guettant le rayon vert sont tour à tour vouées à l'échec par la mouvance continuelle des facteurs impondérables d'une météo hostile, impossible à vaincre par les raisonnements ingénieux : d'une soirée à l'autre, « le ciel aurait fait le désespoir d'un astronome des observatoires d'Edimbourg ou de Greenwich. Il était comme ouaté d'une sorte de vapeur [...]. Lunettes ou télescopes des plus puissants modèles, le réflecteur de Cambridge tout comme celui de Parsontown, ne seraient pas parvenus à la percer» (Verne, 2005, p. 97 ; cf. p. 138).

Comme le rayon est un phénomène optique, ce sont surtout les « vapeurs » couvrant l'azur et entravant la visibilité (brume, brouillard, nuages) qui constituent l'obstacle principal ${ }^{15}$. Ces météores dits « aqueux » par l'ancienne science (par opposition aux météores « ignés » tel que le rayon vert) présentent, dans la langue française, des connotations négatives dues à leur étymologie, fait que Verne exploite à fond. Le terme « brouillard» comporte l'idée de bouillonnement, de confusion, de trouble ; celui de nuage accentue l'aspect du voile et de la couche. Les deux mots évoquent donc un ciel plus ou moins caché, oscillant entre le visible et l'invisible - une dialec-

\footnotetext{
${ }^{14}$ Voir aussi le site déjà indiqué de Jacques Crovisier https://lesia.obspm.fr/perso/jacques-crovisier/ $\mathrm{JV} /$ verne_gene.html qui contient de nombreuses références bibliographiques.

${ }^{15}$ Pour le paragraphe suivant voir Vignes, 2005. Cf. aussi Becker, 2018, p. 109-137 ; Becker, 2012b, p. 57-120 ; Becker \& Leplatre, 2014, p. 11-56.
} 
tique qui est le fil conducteur du récit. Abondent alors les " brumeux crépuscules » (Verne, 2005, p. 35), les « vapeurs dont se chargeait l'horizon » (Verne, 2005, p. 98), les « nuages aux formes peu accusées» (Verne, 2005, p. 217) qui « tamisaient les rayons solaires » (Verne, 2005, p. 103), bref : « ce n'était plus que ciel incertain, nuages orageux, horizons sillonnés d'éclairs de chaleur, bruines crépusculaires » (Verne, 2005, p. 138). Face à cette réalité décevante, les protagonistes ne perdent pourtant pas l'espoir : «Une trouée pouvait se faire dans la basse zone des nuages, une fente, un hiatus, de quoi laisser passer le dernier rayon!» (Verne, 2005, p. 160). Ils s'accrochent à leur rêve d'un temps idéal, « un beau temps » (Verne, 2005, p. 35), où « le soleil brillait d'un vif éclat, et le ciel étendait au-dessus de la mer son azur d'une limpidité parfaite » (Verne, 2005, p. 99 ; cf. p. 130) - un événement si rare qu'il devient presque une illusion.

Toutes ces observations sont accompagnées par des commentaires scientifiques souvent très détaillées, puisés sans aucun doute dans les publications des érudits de l'époque, bien que les traités de météorologie manquent dans la bibliothèque bien fournie de Jules Verne (Burgaud, 1996, p. 129-136) ${ }^{16}$. Ces exposés méthodiques portent par exemple sur la formation des nuages : « des nuages en général, de leur mouvement descendant qui les ramène à l'horizon avec l'abaissement de la température, des vapeurs réduites à l'état vésiculaire, de leur classement scientifique en nimbus, stratus, cumulus, cyrrus » (Verne, 2005, p. 98 ; cf. p. 188-190) ; sur les mécanismes du cycle de l'eau : «Par une sorte de circulation incessante [...], il [l'océan] se nourrit lui-même avec les vapeurs qu'il émet, dont il alimente les sources, qui lui reviennent par les fleuves, ou qu'il reprend directement par les pluies sorties de son sein » (Verne, 2005, p. 151) ; mais aussi sur les différentes hypothèses (aujourd'hui réfutées) expliquant la couleur verte du rayon : «parce qu'au moment où il traverse la mince couche d'eau il s'imprègne de sa couleur [...]. À moins que ce vert ne succède tout naturellement au rouge du disque, subitement disparu, mais dont notre œil a conservé l'impression, parce que, en optique, le vert en est la couleur complémentaire » (Verne, 2005, p. 179).

Le lecteur est également confronté à des analyses climatiques méticuleuses (« Zéro vingt et un d'oxygène, et zéro soixante-dix-neuf d'azote, avec un peu de vapeur d'eau, en quantité hygiénique. Quant à l'acide carbonique, à peine quelques vestiges », Verne, 2005, p. 82-83) et à des bulletins météorologiques très exactes ( « on annonce que la dépression de Swinemunde a marché vers le nord en se creusant sensiblement. Son centre est aujourd'hui près de Stockholm, où le baromètre, en baisse d'un pouce, soit vingt-cinq millimètres - pour employer le système décimal en usage chez les savants - marque seulement vingt-huit pouces et six dixièmes, soit sept cent vingt-six millimètres », Verne, 2005, p. 81 ; cf. p. 216). En fait, la vie quotidienne

${ }^{16}$ Ainsi, Daniel Compère ne mentionne aucun renvoi précis (Compère, 1996, p. 137-146), tout comme Christian Robin qui ne réussit pas à repérer « une source directe » du romancier (Robin, 2008, p. 58). 
des protagonistes est déterminée par la consultation du baromètre, d'ailleurs un « excellent baromètre anérö̈de » (Verne, 2005, p. 98), qui devient le symbole de leur inquiétude (Verne, 2005, p. 38, 81, 98, 103, 108, 130, 142, 187, 216). Cependant, tous ces développements scientifiques, promettant quelque orientation dans des conditions atmosphériques peu propices, sont, eux aussi, profondément minés par l'ironie du romancier, qui soumet le discours rationnel à la même critique narquoise que les autres expressions de ce concert de voix sur le temps qu'il fait, lequel constitue la base même du roman.

Car les expertises données sur la météo sont mises dans la bouche d'un scientifique qualifié franchement de « ridicule » (Verne, 2005, p. 81) et décoré du nom significatif et douteux Aristobulus Ursiclos, qui sent les « livres d'opéra-bouffe » (Pourvoyer, 1990, p. 34). Véritable « cryptogramme », « rébus à résoudre » (Sadaune, 1999, p. 20), ce patronyme associe « la référence savante à Aristote et à l'Encyclopédie (,siclos"), et l'allusion à la maladresse (,ursus" = ours), au discours creux (,bulle”) et au pédantisme (l'apparence latine) » (Compère, 2005, p. 53). Ce personnage-clé de l'intrigue, dont les conseils guident les membres de l'équipe, tout en détruisant, à plusieurs reprises, leur réussite finale par ses interventions étourdies, représente « une bien piètre incarnation de la science » (Pourvoyeur, 1990, p. 35), un exemplaire très particulier des nombreux « savants fous » qui peuplent l'œuvre de Jules Verne (Laszlo, 1996, p. 117-128). Son portrait est des plus dénigrants, voire farouches :

Trop instruit pour un jeune savant, qui ne sait qu'ennuyer les autres de son instruction universelle, gradué des Universités d'Oxford et d'Édimbourg, il avait plus de science physique, chimique, astronomique et mathématique que de littérature. Au fond, très prétentieux, il ne s'en fallait de presque rien qu'il ne fût un sot. Sa principale manie, ou sa monomanie, comme on voudra, c'était de donner, à tort et à travers, l'explication de tout ce qui rentrait dans des choses naturelles; enfin une sorte de pédant, de relation désagréable (Verne, 2005, p. 77).

«Véritable caricature du discours didactique avec sa manie de vouloir tout expliquer, son ton impérieux et pédagogique, sa volonté de sérieux » (Compère, 2005, p. 53), il représente « un scientifique, pour qui tout est chiffre, comptes, mesures, formules, statistiques » (Picot, 1992, p. 66) et qui n'appréhende le monde qu'à travers le filtre de ses lectures universitaires : « les Pascal, les Newton, les Laplace, les Arago, les Humphrey Davy, les Edison, les Pasteur, etc. »(Verne, 2005, p. 87). L'ultime conséquence de cette déformation professionnelle consiste à vouloir publier des mémoires sur les sujets complètement absurdes que lui propose Olivier, sans s'apercevoir du sarcasme inhérent à ses suggestions, dont celle-ci : «De l'influence des instruments à vent sur la formation des tempêtes » (Verne, 2005, p. 180).

Le blâme jeté sur ce personnage vise surtout l'absence de sensibilité, le manque d'humanité : «Il n'écoutait pas, il ne voyait rien, il ne se taisait jamais » (Verne, 
2005, p. 78). «Ursiclos, c'est la science faite homme, l'explication scientifique prioritaire, desséchante et inhumaine » (Pourvoyeur, 1990, p. 36) : « son aveuglement rare envers les sentiments humains » (Derivery, 1994, p. 21) en fait un individu diminué, " hors d'état d'appréhender autrui en termes d'affectivité » (Picot, 1992, p. 66), qui « a perdu tout sens moral » de sorte qu'il déchoit en « misanthrope » et en « macho misogyne » (Laszlo, 1996, p. 120 et p. 122). S'il est le mari choisi pour Helena par ses oncles, cette union s'avère a priori impossible, car elle opposerait deux êtres totalement « antipathiques, séparés par l'abîme qui se creuse entre la vulgaire prose et la sublime poésie, l'un avec sa manie de tout réduire à des formules scientifiques, l'autre ne vivant que dans l'idéal» (Verne, 2005, p. 181). Helena lui reproche violemment son attitude : « Avoir dépoétisé son rayon, avoir matérialisé son rêve, avoir changé l'écharpe d'une Valkyrie en un brutal phénomène d'optique !» (Verne, 2005, p. 181). À travers son regard scientiste et rationaliste, « la nature et le réel, y compris les émotions humaines, sont démétaphorisés, désubjectivés, dés-affectivés »; il manque surtout de « tout ce qui procède de la sensation, de la sensualité » (Picot, 1992, p. 66 et p. 70).

Helena, en revanche, savoure la nature, et notamment la pluie et le beau temps, avec tous les sens : au moment de la tempête qui marque le point culminant de l'intrigue vers la fin du roman, le narrateur note : «Cette existence, sur un îlot désert, fouetté par la tempête, allait à sa nature ardente " (Verne, 2005, p. 228). Cette « météo-sensibilité $»^{17}$, qui relève de ce que le $\mathrm{XIX}^{\mathrm{e}}$ siècle nomme « cénesthésie $»^{18}$, la pousse à s'aventurer toute seule dans la célèbre grotte de Fingal malgré - ou plutôt en raison - de l'ouragan et de la marée montante, cherchant à s'exposer, corps et âme, à la violence des éléments déchaînés par un " enthousiaste et téméraire désir » (Verne, 2005, p. 231). La fureur des météores ${ }^{19}$ suscite en elle des troubles physiologiques et des émotions très vives, qui sont les réactions d'un individu volontairement soumis à la puissance d'une nature sublime : «Je n'ai pas peur... non ! [...] puis-je avoir un autre sentiment que celui de l'admiration devant un tel spectacle! » (Verne, 2005, p. 241). Le danger couru ne provoque en elle que joie et extase : « Miss Campbell était souriante, et toute à la sublimité de ce spectacle » (Verne, 2005, p. 242). À un second degré, cet abandon voulu aux forces de la nature évoque bien sûr l'éruption d'une sexualité refoulée, et le sauvetage dramatique de l'héroïne par Olivier est un chiffre transparent de l'acte charnel : «Olivier Sinclair s'était relevé. Sa résolution

${ }^{17}$ L'expression remonte à Martin de La Soudière (La Soudière, 1999, p. 340). Voir Corbin (Ed.), 2013 ; Reichler, 2012, p. 213-236 ; Ramponi, 2015, p. 39-56.

${ }^{18}$ Le terme coenaesthesis (terme composé à l'aide du grec koinos « commun », et aisthêsis « sensibilité, perception ») est développé par le médecin allemand Johann Christian Reil en 1794. Il s'agit de la traduction artificielle du terme allemand Gemeingefühl (« sensation partagée »), lui-même forgé par Reil. Le terme sera largement adopté par les savants et écrivains du XIX ${ }^{\mathrm{e}}$ siècle. Voir Vigarello, 2014, p. 124-132.

${ }^{19}$ Pour l'importance du motif de la tempête voir les contributions du volume Le Roy Ladurie, Berchtold \& Sermain (Eds.), 2007. 
de pénétrer dans la grotte n'avait pas faibli. [...] Miss Campbell, épuisée, ses forces l'abandonnant, fut prise de défaillance » (Verne, 2005, p. 234, p. 245). Les éléments naturels ${ }^{20}$ symbolisent la passion érotique d'une façon si évidente que l'on s'est posé la question du fond autobiographique de cette scène : «Que s'est-il donc passé dans la vie de Verne, lors de ce voyage dans les Hébrides? » (Derivery, 1994, p. 26).

Ce retour à la nature, qui a parfois été qualifié d' « évasif» et de « régressif $»^{21}$, restitue donc aux météores toute leur valeur sensorielle et phénoménologique (Schneider, 2010, p. 99-120) : contrairement à Aristobulus Ursiclos et sa manie de tout expliquer, Helena « dédaigne les causes et se contente des impressions » (Verne, 2005, p. 181). L'émerveillement résultant d'un « temps vécu» (La Soudière, 1999, p. 30) correspond donc à un refus du désenchantement du monde pratiqué par l'esprit scientiste et à une volonté décisive de rendre à la nature son pouvoir de fascination. Telle est l'intention explicite d'un dernier discours sur les météores que Jules Verne ajoute au roman dans une ultime pirouette : celui de l'artiste. Olivier Sinclair, qui est peintre et alter ego du romancier, favorise une interprétation esthétique des phénomènes atmosphériques, de sorte que l'art devient le vrai mode d'appréhension du monde naturel dans sa complexité et ses énigmes : un postulat qui « corrige » en quelque sorte les deux extrêmes inconciliables d'une perception sensorielle et émotionnelle, et d'une analyse rationnelle et objective (Dehs, 2005, p. 264) 22 .

Olivier se propose d'imiter, grâce à son pinceau, le « sens artiste de la nature » (Verne, 2005, p. 224), et il expose, dans le chapitre-annexe appelé « conclusion » (Verne, 2005, p. 259) par Verne, une toile (un « coucher du soleil ») représentant le rayon vert, dont il saisit intuitivement tout le pouvoir (car lui-même, trop occupé par les yeux d'Helena, ne l'a pas vu). L'artiste, le peintre comme l'écrivain, relève ainsi le défi de capter l'éphémère et de saisir la beauté transitoire, il réussit à fixer l'impression d'un instant et à immortaliser une vision fuyante ; et les admirateurs du tableau exposé par Olivier constatent, et avec « raison », que « mieux vaut regarder le Rayon-Vert en peinture ... qu'en nature » (Verne, 2005, p. 261). Ce passage poétologique, une sorte de manifeste métalittéraire thématisant, dans une mise en abyme, la conception de l'art telle que la réalise le roman de Jules Verne lui-même, n'est pourtant pas exempt de l'ironie constitutive du récit entier, car l'écrivain fait finalement un clin d'œil amusé à son public fidèle, contraint à réviser ses habitudes de lecture face à l'œuvre d'un auteur à multiples talents.

\footnotetext{
${ }^{20}$ Pour le symbolisme de la grotte cf. Becker \& Leplatre, l'article « Souterrain » (sous presse).

${ }^{21}$ Ainsi, Friedrich Wolfzettel voit dans la catabase dans la grotte un retour fusionnel dans la matrice de la Terre-mère, équivalent à une descente dans les profondeurs de l'âme (Wolfzettel, 1988, p. 73-74). Voir aussi Derivery, 1994, p. 30.

${ }^{22}$ Voir aussi Y. Le Scanff qui insiste sur cette " motivation méta-romanesque du récit vernien » (Le Scanff, 2007, p. 255) ; ainsi que F. Raymond qui note que Verne cherche ici à « parler de son art en poète, voire en théoricien » (Raymond, 1994, p. 103).
} 


\section{BIBLIOGRAPHIE}

Becker, K. (2012a). Discours météorologiques et discours littéraires en France, du Moyen Âge à l'époque contemporaine. In K. Becker (Ed.), La Pluie et le beau temps dans la littérature française. Discours scientifiques et transformations littéraires, du Moyen Âge à l'époque moderne (pp. 15-56). Paris : Hermann.

Becker, K. (2012b). La fascination du nubigène. Les discours sur les nuages dans la littérature française. In K. Becker (Ed.), La Pluie et le beau temps dans la littérature française. Discours scientifiques et transformations littéraires, du Moyen Âge à l'époque moderne (pp. 57-120). Paris : Hermann.

Becker, K. (2016). La symbolique du feu et de la flamme dans la littérature. Linguae \&. Rivista di lingue e culture moderne, 15/1, 9-28. Retrieved from http://www.ledonline.it/index.php/linguae/article/ view/959.

Becker, K. (2018). Les vapeurs des écrivains : les nuages et le brouillard dans la littérature française du $\mathrm{XIX}^{\mathrm{e}}$ siècle. In P. Glaudes \& C. Klettke (Eds.), Nuages romantiques. Mise en fiction et traitement esthétique (pp. 109-137). Berlin : Frank \& Timme.

Becker, K. \& Leplatre, O. (2014). Introduction. La brume et le brouillard - deux « météores » énigmatiques dans l'histoire de la science, de la littérature et des arts. In K. Becker \& O. Leplatre (Eds.), La brume et le brouillard dans la science, la littérature et les arts (pp. 11-56). Paris : Hermann.

Becker, K. \& Leplatre, O. (in press). Souterrain. In Ph. Cométy \& J. Lamy (Eds.), Dictionnaire Littérature et nature.

Belleguic, Th. (2003). Diderot et le temps qu'il fait : portrait de l'écrivain en météorologue. Tangence, 73, 9-37.

Belleguic, Th. \& Vasak, A. (2013). Introduction. « Météore » : brève enquête sur le mot et la chose. In Th. Belleguic \& A. Vasak (Eds.), Ordre et désordre du monde. Enquête sur les météores, de la Renaissance à l'âge moderne (pp. 1-26). Paris : Hermann.

Bonn, K. (2014). Vom grünen Leuchten, vom grünen Licht und vom Zufall - Jules Verne, Eric Rohmer und F. Scott Fitzgerald. Philologie im Netz, 70. Retrieved from http://web.fu-berlin.de/phin/phin70/ p70t1.htm.

Burgaud, Ph. (1996). La bibliothèque scientifique de Jules Verne. In D. Jacquart (Ed.), De la science en littérature à la science-fiction (pp. 129-136). Paris : Éditions du CTHS.

Burke, E. (1792). A Philosophical Inquiry Into the Origin of Our Ideas of the Sublime and Beautiful. Basil : J.J. Tourneisen.

Chevrier, A. (1999). La « Folie du vert » : un cas de vision monochrome chez J. Verne. Bulletin de la Société Jules Verne, 131, 25-29.

Compère, D. (1996). Le jeu avec les références scientifiques dans les romans de Jules Verne. In D. Jacquart (Ed.), De la science en littérature à la science-fiction (pp. 137-146). Paris : Éditions du CTHS.

Compère, D. (2005). Jules Verne, parcours d'une ouvre. Amiens : Encrage.

Compère, D. (2013). La Science romanesque de Jules Verne. Amiens : AARP - Encrage édition.

Corbin, A. (Ed.) (2013). La pluie, le soleil et le vent. Une histoire de la sensibilité au temps qu'il fait. Paris : Aubier.

Crovisier, J. (2005). L'astronomie de Jules Verne. In Ph. Mustière \& M. Fabre (Eds.), Jules Verne. Les Machines et la Science (pp. 66-73). Nantes : Coiffard.

Dehs, V. (2005). Jules Verne. Eine kritische Biographie. Düsseldorf und Zürich : Artemis \& Winkler.

Derivery, F. (1994). Pulsions et inscriptions dans deux romans de Jules Verne. Paris : E.C. Éditions.

Dufour, L. (1982). Météorologie et littérature - Le rayon vert dans la littérature française. Ciel et Terre, $98,122-123$.

Dupuy, L. (2013). Jules Verne : la géographie et l'imaginaire. Aiglepierre : La Clef d'Argent. 
Evans, A.B. (1996). Literary Intertexts in Jules Verne's Voyages Extraordinaires. Science Fiction Studies, vol. 23 , no. $69,171-187$.

Frappa, É. (2019). Jules Verne et le mystère du rayon vert. Bulletin de la Société Jules Verne, 198, 77-89. La Soudière, M. de (1999). Au bonheur des saisons. Voyage au pays de la météo, Paris : Grasset.

Laszlo, P. (1996). Le savant fou chez Jules Verne. In D. Jacquart (Dir.), De la science en littérature à la science-fiction (pp. 117-128). Paris : Éditions du CTHS.

Le Lay, C. \& Tirard, S. (2011). Le climat sans dessus dessous. In Ph. Mustière \& M. Fabre (Eds.), Rencontres Jules Verne. Science, technique et société (pp. 195-203). Nantes : Coiffard.

Le Roy Ladurie, E., Berchtold, J. \& Sermain, J.-P. (Eds.) (2007). L'Événement climatique et ses représentations $\left(X V I I^{e}-X I X^{e}\right.$ siècle). Histoire, littérature, musique et peinture. Paris : Desjonquères.

Le Scanff, Y. (2007). Le Rayon vert et le problème de la représentation romanesque. In Ch. Reffait \& A. Schaffner (Eds.), Jules Verne ou Les inventions romanesques (pp. 249-262). Amiens : Encrage.

Michel, A. (1980). Jules Verne et la science romantique. In Jules Verne, écrivain du XIXe siècle (2). Jules Verne : filiations, rencontres, influences (pp. 117-123). Paris : Minard.

Picot, J.-P. (1992). Le conteur et le compteur ou Jules Verne entre science et sentiment. In F. Raymond (Dir.), Jules Verne 6. La science en question (pp. 57-83). Paris : Minard.

Porcq, Ch. (1990). Helena Campbell et ses filles, ou les rayons du Rayon vert. Bulletin de la Société Jules Verne, 93, 40-44.

Pourvoyeur, R. (1990). Une autre lecture du Rayon vert. Bulletin de la Société Jules Verne, 93, 33-37.

Ramponi, P. (2015). Wetterfühligkeit und Diätetik. Skizzen zur literarischen Wissensgeschichte eines kulturellen Symptomleidens 1800/1900. In K. Becker, V. Moriniaux \& M. Tabeaud (Eds.), L'alimentation et le temps qu'il fait -Essen und Wetter - Food and Weather (pp. 39-56). Paris : Hermann.

Raymond, F. (1994). Au rendez-vous des artistes : Le Rayon-Vert. In P. Avrane, Ch. Chelebourg, J. Nassif \& F. Raymond (Eds.), Jules Verne 7. Voir du feu. Contribution à l'étude du regard chez Jules Verne (pp. 101-116). Paris : Lettres modernes.

Reichler, Cl. (2012). Météores et perception de soi : un paradigme de la variation liée. In K. Becker (Ed.), La Pluie et le beau temps dans la littérature française. Discours scientifiques et transformations littéraires, du Moyen Âge à l'époque moderne (pp. 213-236). Paris : Hermann.

Robin, Ch. (2008). La description des météores : un haut moment de vulgarisation dans les Voyages extraordinaires. In Ph. Mustière \& M. Fabre (Dir.), Rencontres Jules Verne. Le partage du savoir (pp. 57-64). Nantes : Coiffard.

Roboly, D. (2005). Jules Verne ou la science au service du merveilleux. In Ph. Mustière \& M. Fabre (Eds.), Jules Verne. Les Machines et la Science (pp. 168-175). Nantes : Coiffard.

Sadaune, S. (1999). Jules Verne, des Cinq Cent Millions de la Bégum au Rayon-Vert ou la démythification de l'idée traditionnelle de «progrès ». Bulletin de la Société Jules Verne, 130, 14-21.

Sadaune, S. (2004). Les 60 «Vyages extraordinaires » de Jules Verne. Rennes : Ouest-France.

Saint Girons, B. (2005). Le Sublime, de l'Antiquité à nos jours. Paris : Desjonquères.

Schaaf, F. (2006). Der grüne Blitz. Astronomie Heute, Dezember, 30-34.

Schneider, S. (2010). Enzyklopädie, wissenschaftliche Beobachtung, Beschreibung. Drei Formen von Objektivität und ihre ironische Vermittlung in Vernes Romanen De la Terre à la Lune und Autour de la Lune. In N. Bender \& S. Schneider, Steffen (Eds.), Objektivität und literarische Objektivierung seit 1750 (pp. 99-120). Tübingen: Narr.

Serres, M. (1994). Atlas. Paris : Julliard.

Vasak, A. (2008). Météorologies. Discours sur le ciel et le climat, des Lumières au romantisme. Paris : Champion.

Verne, J.J. (1973). Jules Verne. Paris : Hachette.

Verne, J. (2005). Le Rayon vert. Paris : Librairie Générale Française. 
Vierne, S. (1973). Jules Verne et le roman initiatique. Contribution à l'étude de l'imaginaire. Paris : Éditions du Sirac.

Vierne, S. (2005). Poétique de la science et de la machine. In Ph. Mustière \& M. Fabre (Eds.), Jules Verne. Les Machines et la Science (pp. 299-306). Nantes : Coiffard.

Vigarello, G. (2014). Le sentiment de soi. Histoire de la perception du corps. Paris : Seuil.

Vignes, C. (2005). Du brouillage à la crise de la représentation : vapeurs, brumes et fumées dans la littérature de la seconde moitié du dix-neuvième siècle. Lille : Atelier national de reproduction des thèses.

Wolfzettel, F. (1988). Jules Verne. Eine Einführung. München und Zürich : Artemis.

Young, A.T. (1998). The green flash. Weather, 53/6, 189-190. 